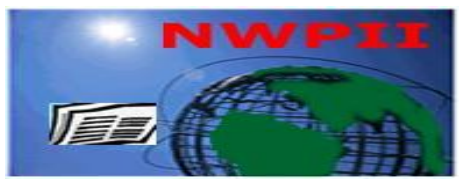

American Journal of Biomedical Sciences

ISSN: 1937-9080

nwpii.com/ajbms

\title{
Epidemiological Studies and Validity of Diagnostic Test
}

\author{
Callixte Yadufashije ${ }^{1}$, Thierry Habyarimana ${ }^{2}$
}

${ }^{1}$ Department of biomedical laboratory sciences, INES-Ruhengeri, Rwanda

${ }^{2}$ Department of biomedical laboratory sciences, INES-Ruhengeri, Rwanda

*Corresponding Author

Callixte Yadufashije

Department of Biomedical Laboratory sciences

INES-Ruhengeri-institute of applied sciences

Rwanda

Email: cyadufashije@ines.ac.rw,cyadufashije@gmail.com

Phone: $+250788273428,+250788259005$

Received:02 October 2018; $\mid$ Revised:24 November 2018; $\mid$ Accepted:07 March 2019

\begin{abstract}
Epidemiology is a basic field of public health and deals with health outcomes in human population. There are main important epidemiological studies used in epidemiology to help epidemiologists and other health workers understand the concept of diseases causation, surveillances, investigation and management. Descriptive epidemiological studies are important in epidemiology and facilitate in frequencies of diseases or other health outcomes and use various variables like age, sex, ethnicity region and others to study diseases frequencies and distribution. Cases control studies are epidemiological research and employ outcome to discover the exposure using retrospective information of participants. Cohort study is also an epidemiological research and uses exposure to measure the outcome. In this study; follow-up was done in both cases and controls. Interventional studies seek to assess the effect of intervention given for a certain outcome. The outcome may be diseases and the investigator can assess the effect of treatment or vaccine. This study is used in clinical trials.
\end{abstract}

Keywords: Cases, Cohort, Controls, Epidemiology

\section{Introduction to epidemiology}

\subsection{Definition}

Epidemiology originated from three important Greek words (epi), which means on or upon, demos, which means people, and the last is logo, which means the study of or the science of. Analytically, epidemiology understood as the science dealing with what is going on within human population.
Definition can be elaborated but as epidemiology deals with human population, we can take the below definition in public health aspects of epidemiology [1].

Epidemiology is the study or sciences dealing with distribution and determinants of health-related events in specified human populations, and its applicability aimed to control health related matters to have healthy population. 
Epidemiology is known to be a scientific discipline that uses scientific methods to investigate health issues in human population. Epidemiology is a quantitative science that uses data and systematic collection approaches to avoid biased results. Basically epidemiology methods aimed on serious observational methods and utilize comparison methods between groups of population to measure exposure and outcomes in population ${ }^{[2]}$.

Generally, epidemiology can be described as a fundamental discipline of public health for positive health of people. First, epidemiology is considered to be quantitative science that basically relies on a working mathematical knowledge like probability, statistics, and may explain early research methodology of clinical researches in medical sciences. Secondly, epidemiology can be known as a method leading to the cause of elaborating many reasons based on the development and hypothesis tests in sciences like biology, physics, behavioral sciences and ergonomics to provide detailed explanation on health related events, behavior and state $^{[1-2]}$.

Distribution: Epidemiology deals with the frequency and pattern of health events in human population: Frequency tends to explain not only the existing number of health events like the number of cases of pneumonia or cancers in a population, but also deals with the relationship between the mentioned numbers of cases of a certain outcome to the total population reside in a considered region. This leads to results that support ideas of epidemiologist in comparing the occurrence of diseases in different population ${ }^{[3]}$.

Pattern is known to deal with the occurrence of health-related events or state by consideration of time, place, and person. Time patterns can be understood in terms of year, seasonal, weekly, daily, and hourly and other time that are not mentioned here but that can contribute to diseases, disabilities and injury are considered. Place patterns are counted in terms of geographical variation, urban regions/rural regions differences, and residences of work places or schooling sites. Individual characteristics including demographic factors which may have correlation to risk of illness, injury, disability, age, sex, marital status, and socioeconomic status, as well as behavioral matters and environmental exposition. These health patterns are constructive elements of descriptive epidemiology ${ }^{[4]}$.

Determinants: Epidemiology is also a good tool to be used in discoveries of determinants, which are known to be the main causes and other risk factors leading to the occurrence of diseases and other health-related problems. Epidemiologists accept that diseases cannot occur randomly in human population, but diseases and other health problems occur when people exposed to determinants or risk factors that favor the occurrence of any health outcome ${ }^{[5]}$. To discover these determinants of diseases, epidemiologists utilize analytic epidemiology leading to responses of "Why" and "How" the disease or other health related matters happen. The assessment of diseases rates in groups of people is done and may differ according to demographic characteristics, immunity level, genetic, behaviors, environmental exposures that are known to be potential risk factors ${ }^{[1-5]}$.

\section{Health-related states or events:} Traditionally, epidemiology was to deal with epidemics of communicable diseases later, modern epidemiology was expanded and dealt with endemic communicable diseases and non-communicable infectious diseases. In 20th Century, the development of new methods of epidemiology has taken place and added applications of epidemiology in chronic diseases, injuries, birth defects, maternal and child health, environmental health and the health issues related to working sites. Thus epidemiologists began looking at behaviors that can have an impact to health and well-being of population ${ }^{[6]}$.

Specified populations: Although epidemiologists and clinicians are both responsible with the control of disease and what is different is the way of approaching the patient. For the clinicians, they only look at the individual health, while epidemiologist considers the health of the community as a whole. Epidemiologists consider collective health for elaboration of mass control of the diseases in the community. Clear understanding is that clinician's patient is individual but the patient for epidemiologists is the community [7]. Epidemiologists and clinicians are all needed and important for health improvement and differ in their responsibilities while treating a patient. For example, once a patient with diarrheal disease 
comes, both epidemiologist and clinician are responsibly interested in conducting the correct diagnostic test. The clinician's task is relying on treatment and care of the patient, while the epidemiologist focuses on the identification of the exposures or sources that caused the disease, and also considers other people in the community who have been exposed to similar risk factors with the patient to prevent the spread of the diseases in the community ${ }^{[8]}$.

Applicability: Epidemiology is not directly the science dealing with health in population, as it involves the application of the knowledge gained through studies to community-based practice. Practical skills in medicine give capacity of medicine to be a science and art, likewise practical skills in epidemiology help epidemiology be among sciences and art. Conducting the right diagnosis and treatment requires the combination of medical or scientific knowledge, judgment done clinically, and understanding of the patient. Epidemiologists use methods that are scientific in descriptive and analytic epidemiology, epidemiologic judgment and understanding the community ${ }^{[2-8]}$.

\subsection{Types of epidemiological studies}

Descriptive epidemiology is among important basic types of epidemiological study, which is responsible in describing the frequency (occurrence) and distribution of diseases and other health related matters in consideration of time, place, and person. The branch of epidemiology dealing with determinants or causes is known as analytic epidemiology and is known to ask question of how and why? ${ }^{[9]}$

\subsubsection{Variables in Descriptive Epidemiology}

The main known variables in descriptive epidemiology can be categorized in: person, place and time. To describe the occurrence of a disease in human population, descriptive epidemiology asks the following questions as well as answers: Who is affected by the disease? Where and when do the diseases occur? ${ }^{[10]}$

\section{Descriptive Epidemiology}

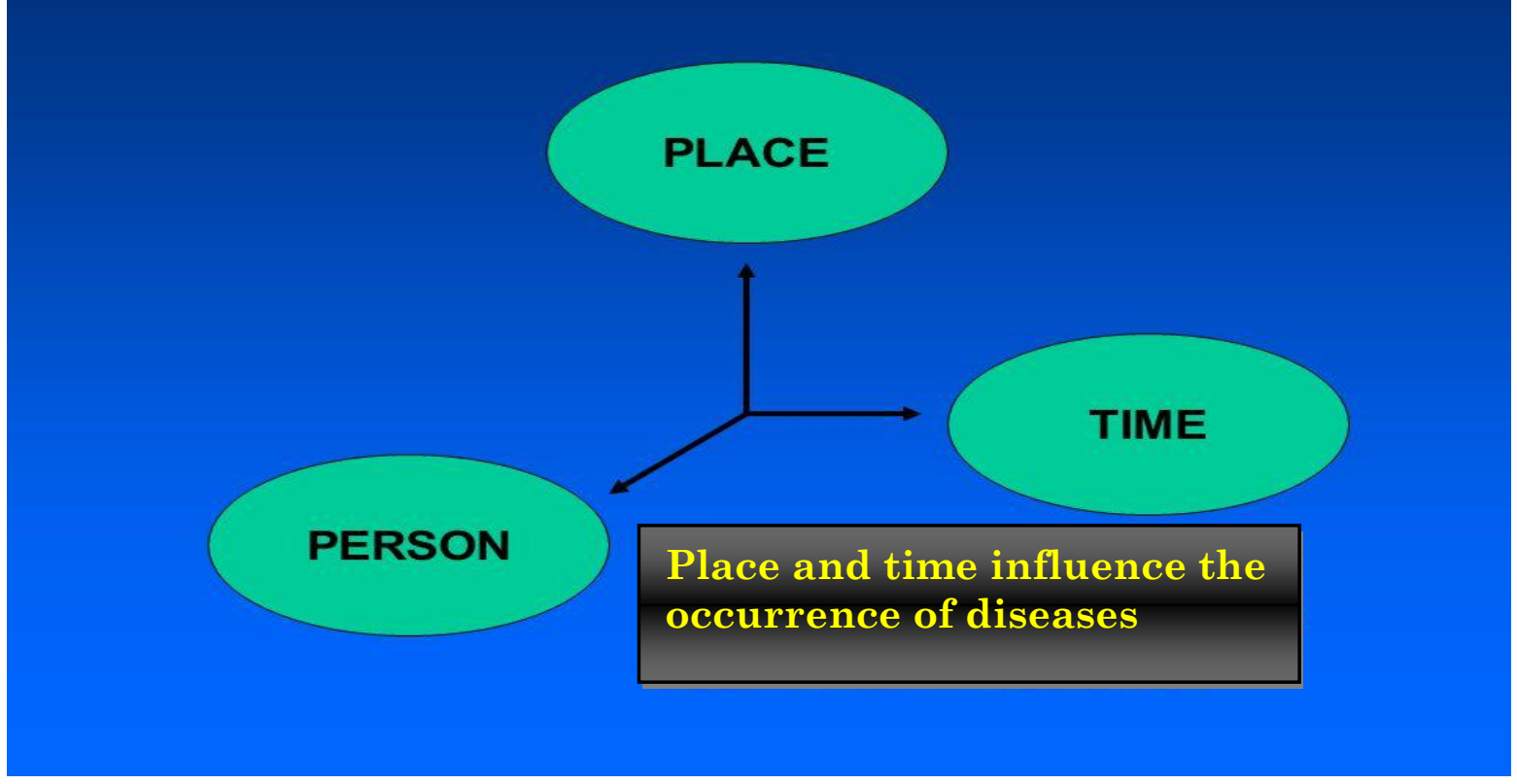

Figure 1: summarizing variables of interest in descriptive epidemiology 
Person: In descriptive epidemiology people are most considered and have various variables to look at for clear understanding of what is taking place in population. Here we characterize person basing on variables like age, sex, ethnicity or race.

Age: Age is very and most useful determinant in personal variables. Example: Measles affect only children.

Sex: There are diseases that are common in men but that are not seen in women. For example, prostate cancer is classified in NCDs affecting only male.

Ethnic group or Race: some disease can affect people according racial groups where you find that frequency is different among ethnic group ${ }^{[3-10]}$.

Place: Disease frequency differs in different regions and places. These differences in disease frequencies can be due to natural boundaries like rivers, mountains and other physical features. Physical features may influence some climate characteristics like temperature, humidity, rainfall and others and these may influence seasonal diseases. For example, Malaria is concentrated in low altitudes but relapsing fever is commonly found in high altitude regions ${ }^{[1-10]}$.

Time: Descriptive epidemiology uses time to study disease occurrence in human population as some diseases may occur monthly or annual basis. There are some diseases that occur periodically or cycles. These Cycles may be annual or have some other periods that go like cycles in time. Seasonal change is known to be an important period in epidemiological studies ${ }^{[8-10]}$.

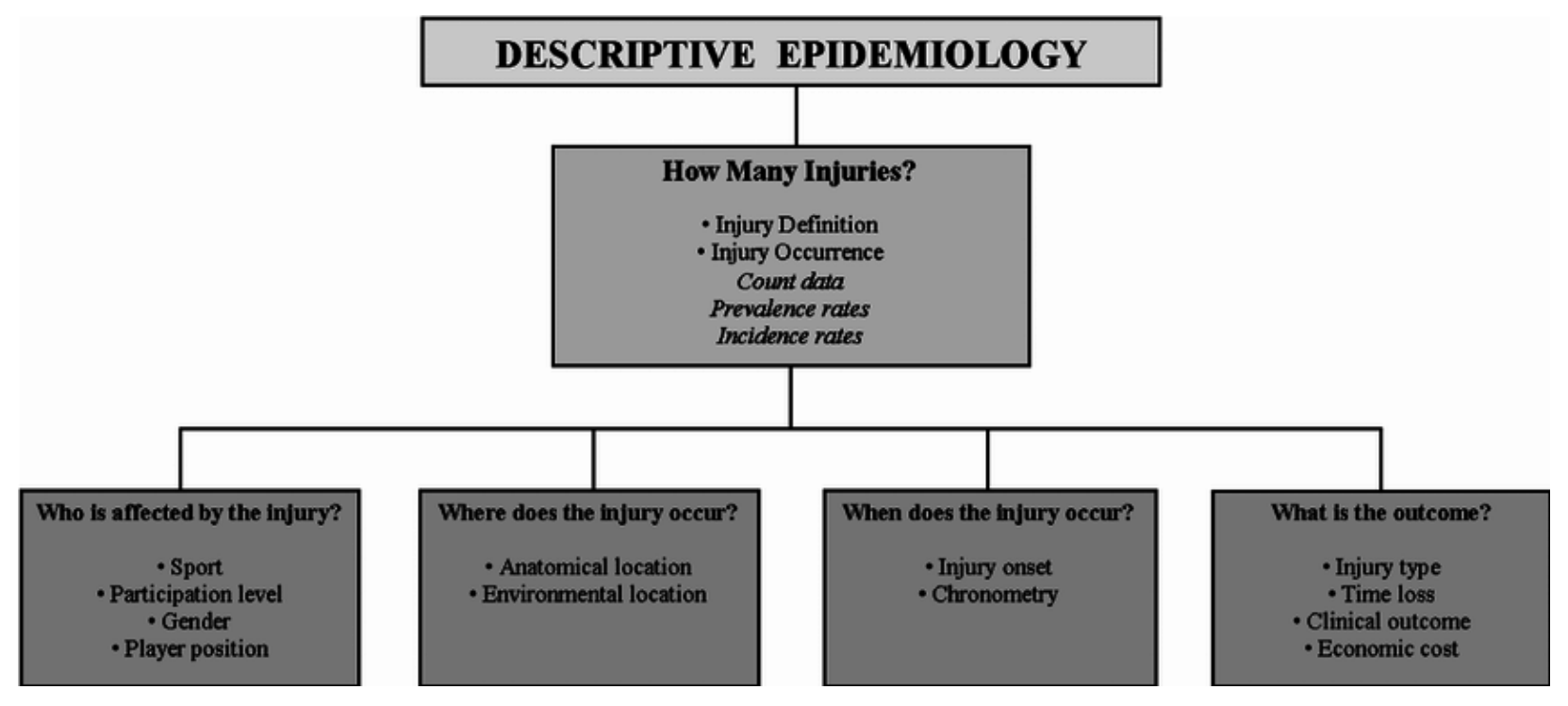

Figure 2: summarizes descriptive epidemiology

\subsubsection{Previous studies on Descriptive epidemiology}

The Descriptive Epidemiology of Female Breast Cancer: An International Comparison of Incidence, Survival and Mortality.

Descriptive epidemiology as discussed early in this chapter, considers the variables: person, place and time. The study was conducted by Z. Zaidi, MD and $\mathrm{M}$. Hamdi Cherif, $\mathrm{PhD}$ at the university Hospital of Setif, Setif, Algeria. In descriptive epidemiology we quantify cases basing on person his location and time. The aim of the study was to compare incidence, survival and death due to breast cancer; these are based on statistical data (numbers).

Methodologically, retrospective data was collected where data regarding the incidence and mortality statistics were collected from International Agency for Research on Cancer: The Cancer Incidence in five Continents Vol IX. GLOBOCAN database, presented on may 2011 and data on cancer survival were collected from Cancer survival in five continents, a worldwide population-based study. Africa is presented by (Setif) Algeria, and also the Cancer survival in Africa, Asia, the Caribbean and Central America (SurvCan). 
The results of the study show cancer as the cancer of high frequency among women where the estimation shows the incidence of 1.38 million new cancer cases diagnosed in 2008. The study continues showing breast cancer as the most common cancer in both developed and developing world with the incidence of 690,000 . The breast cancer survivors are frequently found in developed countries. The results also ranked breast cancer as the fifth cause of death with 458, 0000 deaths. Cancer also was recognized to be the most silent killer in women for both developing and developed regions with 290000 and 180000 deaths respectively. Considering five years collected retrospective data, the estimation shows that Africa has $12 \%$ and $90 \%$ for the United States, Australia and Canada, Saudi Arabia. Considering statistics on survival, developed countries has more done on cancer treatment as the incidence of survival is great done that of developing countries.

Epidemiological Factors in Prevalence of Malaria Parasites in Primary Health Facilities Attendees, Ogun State, Nigeria

This study was conducted by Wobo SO et al in Nigeria and the study period is October, 2012 and January 2013. Methodologically, oral interviews were conducted to collect data from sampled participants of target population. Seven primary health centers were selected and from there 384 consented participants were taken for blood sample collection. Giemsa staining techniques was used for Blood film examination for detection of parasites of malaria in blood sample. Findings showed that among 384 participants tested, 273 which is $71.1 \%$ of total tested participants had malaria parasites according laboratory results.

Malaria is caused by number of Plasmodium species and this test showed Plasmodium falciparum (95.6\%), Plasmodium malariae $(3.3 \%)$, P. ovale $(0.7 \%)$ and P. vivax $(0.4 \%)$. Malaria has been high in children between age-group 1-11 years with prevalence $94(81 \%)$ but in age group 51-60 malaria was low prevalence with $6(54.5 \%)$. The high significance $(\mathrm{p}=0.011, \mathrm{p}<0.05)$ of malaria with age consideration was seen. It has seen that males $110(73.8 \%)$ were more affected comparing to females 163 (69.4\%), however, there was no statistic significance to confirm ( $\mathrm{p}=0.347, \mathrm{p}>0.05)$. it was seen that malaria was prevalently high in less educated people for those without formal education $80(81.6 \%)$ and was shown to be prevalently low among those who completed postsecondary education. Considering occupation, malaria was highly prevalent in unemployed group while least in retired people $(\mathrm{p}=0.014, \mathrm{p}<0.05)$. Those who were not married $(56.4 \%)$ where more affected comparing to those who were married $(43.6 \%)$.

Incidence of prostate and urological cancers in England by ethnic group, 2001-2007: a descriptive study

This study was conducted in 2015 by Mahiben Maruthappu et al and their objective was to comparing the incidence of four types of urological cancers, among those cancers including kidney cancer, bladder cancer, prostate cancer and testicular cancer. This comparison was conducted in non white ethnic group in England and was Indian, Pakistani, Bangladeshi, Black African, Black Caribbean and Chinese and whites. The comparison was done in non whites themselves and non whites to whites. This was a descriptive study and tended to compare incidences. Remember that in descriptive study, ethnic group is variable of importance, this facilitate to understand descriptive epidemiology.

Methodology: During this study, retrospective information regarding ethnicity and urological cancer was considered. It was done through archives registration of urological cancer from 2001 to 2007 ( $n=329,524)$ by linkage to the Hospital Episodes Statistics database. Incidence rate, ratio adjusted was calculated basing on variables like age, sex and income. It was done both six non white ethnic group and whites.

Findings: Generally, the significance difference of incidences in all ethnic groups was pidence rate, ratio adjusted was calculated basing on variables like age, sex and income. It was done both six non white ethnic group and whites. onducted in non white e prostate cancer, which displayed a higher incidence in Blacks. The incidence rate ratio of 2.55 was seen and showed evidences of differences in risk between Indians, Pakistanis and Bangladeshis for kidney, bladder and prostate cancer $(\mathrm{p}<0.001)$, and between Black Africans and Black Caribbeans for all four cancers $(p<0.001)$. 


\subsection{Case-control and cohort studies}

\subsubsection{Introduction}

Case-control and cohort studies are both known as observational studies that are based on the middle of the hierarchy of evidence. Studies of this kind, in combination with randomized controlled trials, are main constituents of analytical epidemiology or analytical studies. On the other side case reports as well as case series make descriptive epidemiology or descriptive studies ${ }^{[11]}$.

\subsubsection{Case-control studies}

Case-control studies are known as retrospective due to its system of using information from the past to study a certain outcome. It is done by defining two important group of interest, where a group with outcome/diseases and the group without outcome get identified ${ }^{[6-11]}$. After identifying these two groups, the following step is looking back for assessing if there is a statistically significant difference in the rates of exposure to the mentioned risk factor between the selected groups. The important measure on this type of study is odds ratio $(\mathrm{OR})^{[12]}$

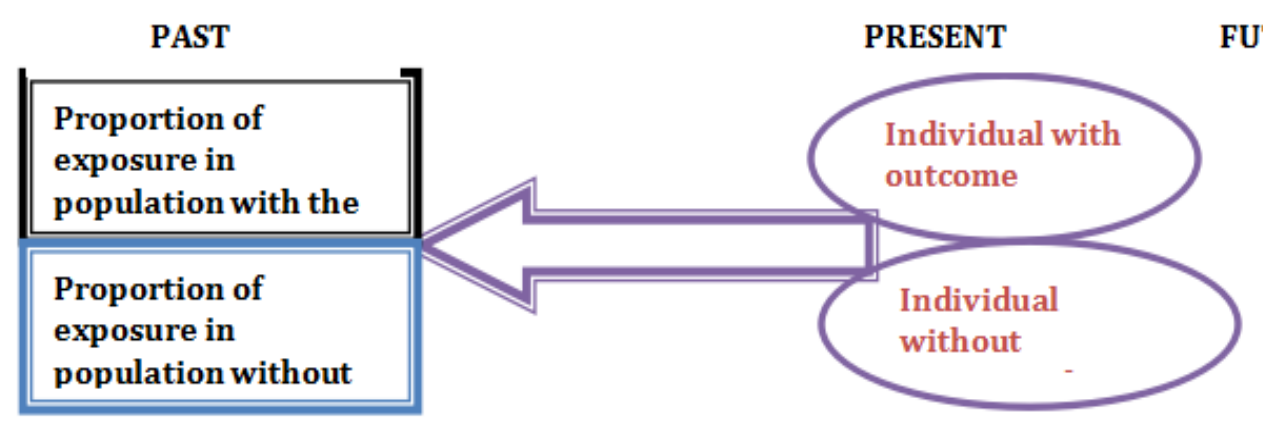

Figure 3: Case-control study design

Case-control studies should include two groups that are identical except for their outcome / disease status.

As such, serious carefulness can be done in selecting controls. Simply controls have to be matched with selected cases considering like age, sex and others to avoid confounding results of a certain study. Case-controls are very fast to provide results and are not costly comparing to other studies. Those types of study use retrospective information; it facilitates the investigator to know diseases that took a long or short time within population of interest. However, it wisely to consider more than one exposure to build better understanding of various risk factors for any considered outcome of interest ${ }^{[13-14]}$. Nevertheless, as case-controls are known to be retrospective, it is important to understand that this type of study is more prone in having results with bias due to its system of giving a freedom to participant to self-report to their exposure on certain outcome. This will require the investigator to pay attention during his investigation depending on who is talking with and also the experience of interview to avoid bias in his results $[15]$

\section{Recent studies on case control:}

(a) The magnitude of the association between smoking and the risk of developing cancer in Brazil: a multicenter study.

This study was done by Moura MA1, Bergmann A, Aguiar SS, Thuler LC. Their objective was the estimation of the degree of association and identification of the fraction of etiology attributable to smoking in development of different types of cancer in Brazil.

Methodology: This was a case control study where 204101 cancer patients registered in Cancer Hospital Registries (CHR) in the period from 1998 to 2011 were considered in the study. This group of patient were compared with 26971 cases of nonmelanoma skin cancer. This comparison is to facilitate the measurement of the exposure as outcome of interest already known. 
Exposures measured at the hospital registration: the exposure of interest measured at at the time of hospital registration is smoking. The researcher calculated odd ratio, unadjusted and adjusted for gender, and age and alcohol consumption, with $95 \%$ CIs.

Findings: After adjustment, the researcher that the risk of the development of cancer is associated with smoking and the association was very high (piriform sinus, bronchi and lung, larynx, hypopharynx, oropharynx and oral cavity), the high association also (oesophagus and bladder), moderate association (anus and anal canal stomach, nasal cavity, middle ear and paranasal sinuses, pancreas, nasopharynx, other parts of the biliary tract and kidney and low association (liver, gall)). The researcher found that there was no association between smoking and cancers of the central nervous system and myeloid leukaemia. The decreased risk of developing was found for thyroid cancer to mean there is no association. For thyroid cancer there was a decreased risk of developing the disease.

Conclusions: the study findings show there is a high risk of developing cancer of the hypopharynx, bronchi and lung, larynx, oropharynx and oral cavity, oesophagus and bladder cancer among smokers and smoking was seen to be the cause of different cancers in Brazil.

(b) Risk Factors for Anemia in Pregnancy: A Case Control Study
The study was conducted in Maharashtra, India by Rutuja Pundkar et al.

Objectives: The overall objective of the study was to find risk factors that lead to anemia in pregnancy. Aim of the study was to find the risk factors leading to Anemia in pregnancy. Specific objectives were to study various sociodemographic factors that lead to anemia and assess the knowledge of study participants on anemia

Methodology: this was a Case control study and was conducted at Primary Health Centre, to determine the risk factors that lead to anemia in pregnancy. The study population was 308 pregnant females who registered during ANC. Using systematic random sampling 2 groups were created to have cases and controls. The group I was for cases and group II was for controls and each group composed by 50 members. For cases were, hemoglobin counts less than $11 \mathrm{gm} \%$ \& for controls was hemoglobin count more than $11 \mathrm{gm} \%$. Laboratory test was conducted and women having hemoglobin less than $11 \mathrm{mg} / \mathrm{dl}$ were considered anemic which means that they were cases. and women with $\mathrm{Hb}>11 \mathrm{mg} / \mathrm{dl}$ were considered controls. Questionnaire for interview also was conducted in two groups to collect sociodemographic information

Results: Findings show that hemoglobin level for controls was $11.55 \mathrm{~g} / \mathrm{dL}$ while it was $9.58 \mathrm{~g} / \mathrm{dL}$ in cases. It seems that there was association between diet, family size, education, social class, gravida and parity with anemia in pregnancy

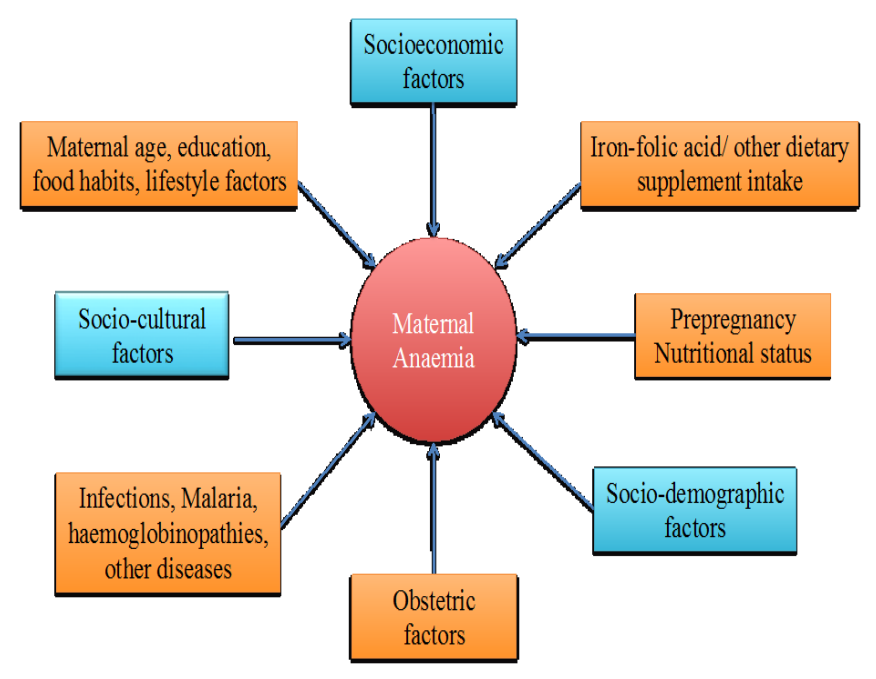

Figure 4: summarizes different factors leading to anemia in pregnancy 
(c) Group B streptococci cultured in urine during pregnancy associated with preterm delivery: a selection problem?

This study was conducted by Mohammed R et al and its objective was to see the investigation of the association between group B streptococci (GBS) in urine culture during pregnancy and preterm delivery.

Methodology: The study has considered a population-based cohort consisted of all the pregnant women $(n=36,097)$ from the study area of Lillebaelt Hospital, Denmark, considering the period January 2002 -December 2012. 34,285 Singleton pregnancies were used and dived in three strata. Stratum I was women whose diagnosis of urine showed positive result for GBS and was 249 $(\mathrm{N}=249)$, group II $(\mathrm{N}=5765)$ was women with urine sample culture showing negative results for GBS, and the last group III $(\mathrm{N}=28271)$ was whose urine had not been cultured during pregnancy. The outcome of importance was preterm birth before 37 weeks' gestation (PTD).

Findings: the study did not find any association between PTB and GBS bacteriuria in considered groups (odds ratios $(\mathrm{OR})=0.89 ; 95 \% \mathrm{CI}$ : 0.5-1.4). After evaluating confounders of interest, the PTB continued to show no association with GBS bacteriuria (adjusted $\mathrm{OR}=0.99$ ). when considering the combination of the cultured groups (I and II), there were association with a statistical significance of higher risk for PTB, when comparing with the group with no urine specimens diagnosed through culture $(\mathrm{OR}=1.96)$ and adjusted or 1.80).

\subsubsection{Cohort studies}

Cohort studies are either retrospective or prospective. The important thing to know is to differentiate retrospective cohort and case control studies. They are totally different. In retrospective cohort studies, both exposure and outcomes have already been identified. They are usually conducted basing on existing data from prospective studies, here the exposure is mentioned before considering the outcome of interest and the outcome will be considered through investigation of susceptible people and then lead to confirmation of acceptance or rejection according to what we have observed to the development of outcome ${ }^{[16]}$.

Prospective cohort studies are more commonly used with many people in health sectors especially medical graduate and others in health care who want to control diseases. During this type of cohort, the recruitment of people in cohort studies has to be done without consideration of exposure or outcome status. The researcher has to set criteria of selection depending on what he is investigating. For example, you think that smoking is a leading cause of lung cancer, your selection will select those who smoke with the outcome or not, and those who do not smoke ${ }^{[17]}$. Follow up will be done to measure the development of outcome in selected groups. What it means here, the study then follows these participants for a limited period of time to assess the proportion of those who develop the outcome of interest $^{[18]}$. Therefore, cohort studies are very good and interesting during assessment of prognosis, risk factors and harm. The measurement of outcome in cohort studies is always a relative risk or risk ratio $(\mathrm{RR})^{[1}$

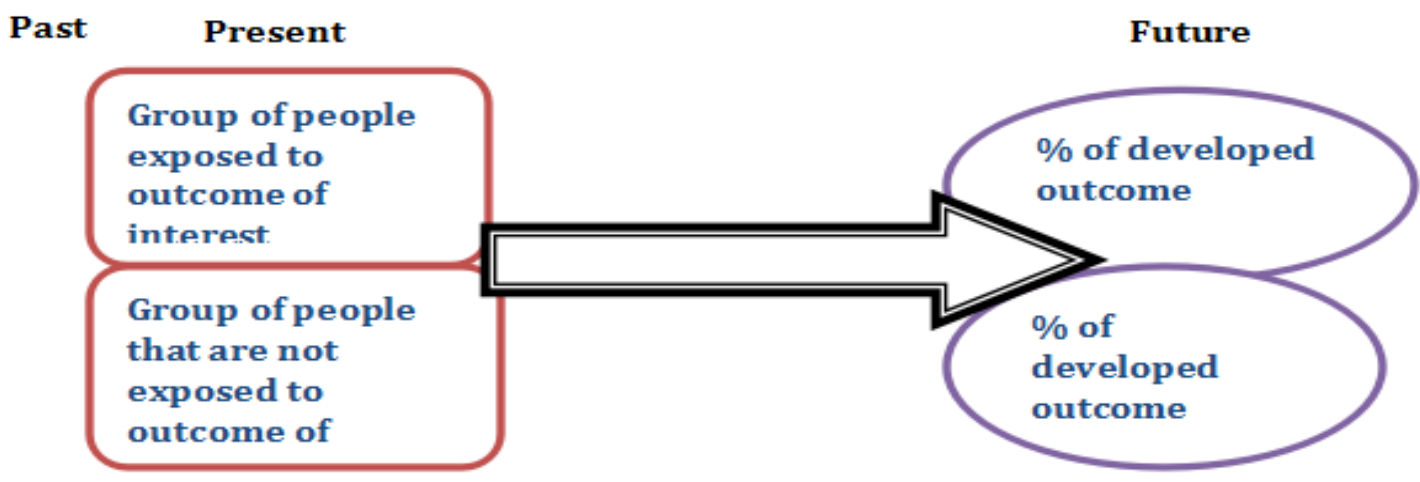

Figure 5: Cohort study design 
Cohort studies should include two groups that are identical except for their exposure status.

As a result, the attention should be done during selection of participants in cohort study. Both exposed and non-unexposed groups should be selected from identical population. After selection, serious follow up should be done and attrition has to be studied carefully to protect validity of the study and this will reduce bias of our results. Cohort studies can assess the proportion of outcomes and this allows an exposure to be discovered and assessed in a simple way and its impact in developing disease is known simply. Thus, cohort studies are significantly important, they are expensive comparing to case control studies depending on considered outcome, period of follow up as well us location ${ }^{[20]}$

\section{Previous studies on cohort study design:}

(a) Retrospective cohort study of smoking and lung cancer incidence in rural prefecture, Japan

The epidemiological study was conducted by Osaki et al., andtended to discover the association between the incidence of lung cancer and smoking habit. In conducting the study, the reference was made to special benefit of smoking cessation and control to reduce the incidence of lung cancer and for promotion of control program in local population.

The study was a retrospective cohort, as scientifically known, in cohort study, exposure has to be seen and follow-up should be done for investigator to see the outcome. During the study, 16,383 males who have been diagnosed for lung cancer in 1995 in Tottori Prefecture, Japan were considered as target population. Questionnaire regarding examination was conducted to measure the exposure variable. Cancer registry was supported to measure the incidence of cancer. Follow up was done for the period of 4.3 years.

Findings showed that current smoker's hazard ratio led to the incidence of lung cancer of 4.9, and for previous smokers was 2.2, showed the determination of dose-relationship between lung cancer and consumption of the cigarette. What findings showed is that the ration was high in young people under 65 years. (b) A prospective cohort study of cigarette smoking and the risk of endometrial cancer

The epidemiological study was conducted by $\mathrm{P}$ D Terry et al. As a prospective study, the aim of the study was to find if there is any association between smoking and endometrial cancer risk in a large prospective cohort. Proportional hazards models were used for the estimation of the hazard ratios associating cigarette smoking to endometrial cancer risk among 70591 women of the age 40-59 years at recruitment into a randomised controlled trial of mammography screening for breast cancer.

The serious follow-up was done averagely in 10.6years in 751833 person-years, and 403 women were tested with incident endometrial cancer. The findings showed that there was a reduction of endometrial cancer risk among women who currently smoked at least 20 cigarettes daily. According to results, suggestion of an inverse association with the duration of smoking was discussed but no evidence to confirm this assertion that has been thought. menopausal status did not show the variability of the association, body weight and hormone replacement therapy also did not show this association, however, the association have been clear among parous than nulliparous women.

(c) Predictors of Obstetrical Complications: A Prospective Cohort Study

The study titled predictors of obstetrical complications was done by Irina et al and its objective was the identification of predictors of obstetrical complications.

Methodology and findings: The prospective cohort studywas conducted at a state-run obstetrical hospital, Omsk, Russia. There were a total of 2,497 pregnant women with a gestation age of less than 12 weeks, and 2,356 women with singlet pregnancies were selected to collect information. Gestation outcome data were available in 2,177 women. The evaluation of social and medical factors was done and blood coagulation screening was done in women whose pregnancy is in the first trimester. Ordered logistic regression was used for the assessment of obstetrical complications risks. Below, these are obstetrical complications of importance that was assessed in 28 weeks of pregnancy: preterm labor, intrauterine growth 
restriction and intrauterine hypoxia, preeclampsia, abruptio placentae,

The results showed that 21 predictors $(\mathrm{P}<0.001)$ have a strong association (0.82) between parameters under studied and the obstetrical complications was seen. $91.1 \%$ of obstetrical complication was seen to be associated with parameters below in women: age, education, parity, medical history, heredity (family history) and blood coagulation profile. The increase of fibrinogen concentrations and the decrease of thrombin clotting time in the first trimester of pregnancy were seen have a high association of severe obstetrical complications late in pregnancy.

\subsection{Intervention studies}

The above described epidemiological studies are observational and this means that they only observe but do not do anything on the outcome of interest. Intervention studies are done by intervening with a group of people, and also include another identical group which is considered to be a "control group" [10-20]. The randomised controlled trial (RCT) is kind of intervention study and is commonly used in intervention studies. RCTs are done by dividing the population of interest into groups randomly, and one group is with treatment or another intervention while the second group is not with treatment or intervention. Investigator should choose people with the same characteristics, and by then, any difference between groups will be due to intervention given or not given ${ }^{\text {21-22] }}$.

It is better for the RCT to be conducted using a double-blind method: that is, neither the researcher nor the subject knows who is in the intervention or control arm of the study, here it has to be understood no need to know whether there is an intervention the researcher will investigate this and discover ${ }^{[22-23]}$.

These types of studies are done in the following way. Initially, observations (or measurements) are made on a population which is then divided (by random sampling) into two equivalent groups. Of these, one is subjected to the intervention like treatment or another intervention done to cure or prevent the outcome. After a while of the intervention, the careful observations are repeated and the results for the two groups are compared to confirm if the intervention brought an effect to the outcome. Drug trials are known to be the most common kind of interventional studies. The purpose of drug trials is discovering the effects and effectiveness of the developed new drugs by the pharmaceutical industry ${ }^{[5-21-23]}$.

\subsubsection{Recent interventional studies:}

(a) A pilot interventional study to evaluate the impact of cholecalciferol treatment on $\mathrm{HbAlc}$ in type 1 diabetes (T1D)

The study was conducted by Perchard et al in 2017 and its main aims were the assessment of the baseline 25(OH)D3 status in a Manchester cohort of children with type 1 diabetes (T1D), the second objective was to determine the effect of cholecalciferol administration on $\mathrm{HbA} 1 \mathrm{c}$.

Methodology and design: The design and methodology considered children with T1D who attended the routine clinic appointments over three months in late winter/early spring and who had blood samples taken with consent. All study Participants who have a 25(OH)D3 level $<50 \mathrm{nmol} / \mathrm{L}$ were given treatment with a one-off cholecalciferol dose of 100,000 ( $2-10$ years) or 160,000 (>10 years) units. The record of HbA1c levels was taken and recorded before and after treatment

Results: The findings showed the level of Vitamin D in 51 children. Among 51 children, there were 35 were Caucasian, 11 South Asian and 5 from other ethnic groups. 42 were vitamin D deficient. All children from South Asian were vitamin $\mathrm{D}$ deficient with mean $25(\mathrm{OH}) \mathrm{D} 3$ of $28 \mathrm{nmol} / \mathrm{L}$. a negative relationship was shown between baseline $25(\mathrm{OH}) \mathrm{D} 3$ level and $\mathrm{HbA} 1 \mathrm{c}$ $(\mathrm{r}=-0.484, \mathrm{P}<0.01)$ among Caucasians. In participants who received treatment, there was no significant difference in mean $\mathrm{HbA} 1 \mathrm{c}$ at 3 months $(\mathrm{t}=1.010, \mathrm{P}=0.328) \quad$ or $\quad$ at 1 year $(t=-1.173, P=0.248)$ before and after treatment.

(b) The effect of educational intervention on knowledge, attitude and glycemic control in patients with type 2 diabetes mellitus

The study was conducted by Marwa et al. and the objective was the assessment of the effect of educational intervention on knowledge and attitudes and glycemic control in type 2 diabetic patients. To reach on results, this interventional study recruited 
100 diabetic patients attending three primary health care units in Port Said City. Patients were put in three health educational session and diabetic information was availed. The assessment on Knowledge, attitude and glycemic control were done before and 3 months after the interventional activities.

Results: The improvement on knowledge and attitude was observed among diabetic patients after implementing educational intervention. The total knowledge mean score increased from $2.69 \pm 1.44$ in the first visit to $5.30 \pm 1.36$ in the second visit, awareness about various aspects of diabetes was marked with patients. There was a high decrease of the mean levels of fasting blood glucose (FBG) and Glycosylated Hemoglobin (HbAlc) at the second visit compared to the first visit $(180.33 \pm 34.81 ; 8.69$ vs. $168.04 \pm 28.56,8.50 \pm 0.97$ respectively). Negative correlation between the knowledge Attitude (KA) score and $\mathrm{HbA1c}$, and between the KA score and FBG was seen.

(c) Effect of a community-based delivery of intermittent preventive treatment of malaria in pregnancy on treatment seeks for malaria at health units in Uganda.

The study was conducted by Mbonye et al and its objective was to assess whether traditional birth attendants, drug-shop vendors, community reproductive health workers and adolescent peer mobilizers can administer IPTp with sulphadoxinepyrimethamine (SP) to pregnant women, reach those at greatest risk of malaria, and increase access and compliance with IPTp.

Design and methodology: This was an interventional study that tended to compare the delivery of IPTp in the community with routine delivery of IPTp at health units. The fraction adolescents and primigravida that were assessed as the first primary outcome measures of interest, and women's proportion who were given two doses of SP was considered in the study for comparison. This study also evaluated interventional effects on accessibility of malaria treatment, antenatal care and other different services.

Findings: The results showed that many women $(67.5 \%)$ received two doses of SP by means of community approach comparing to with health units $(39.9 \% ; \mathrm{P}<0.0001)$. findings also showed that Women who had accessibility on IPTp through the community were at an earlier stage of pregnancy (21.0 weeks of gestation which is the beginning of the second trimester of pregnancy) than women with accessibility on IPTp at health units (23.1 weeks of gestation which later comparing to those of the community; $\mathrm{P}<0.0001)$. although, high fraction of primigravida $(23.6 \%$ vs $20.0 \%$; $\mathrm{P}<0.04)$ and adolescents $(28.4 \%$ vs $25.0 \% ; \mathrm{P}<0.03)$ has visited health units. Commonly, women with accessibility on IPTp at IPTp at health units are them also who made many visits for malaria treatment (2.6 (1.0-4.7) vs 1.8 (1.4-2.2); $\mathrm{P}<0.03)$. During recruitment stage, women with accessibility on IPTp through health units wanted treatment of malaria comparing to those with accessibility on IPTp through community (56.9\% vs $49.2 \%$ ). During delivery, the number of women who accessed IPTp through the community sought malaria treatment $(70.3 \%)$, antenatal care use, insecticide-treated nets, as well as delivery care was higher in the community. Comparing service costs, women who received two doses of SP for IPTp were 4093 Uganda shillings (US\$ 2.3) for women with accessibility on IPTp at health units, while it was 4491 Uganda shillings (US\$ 2.6) for women with accessibility on IPTp through the community.

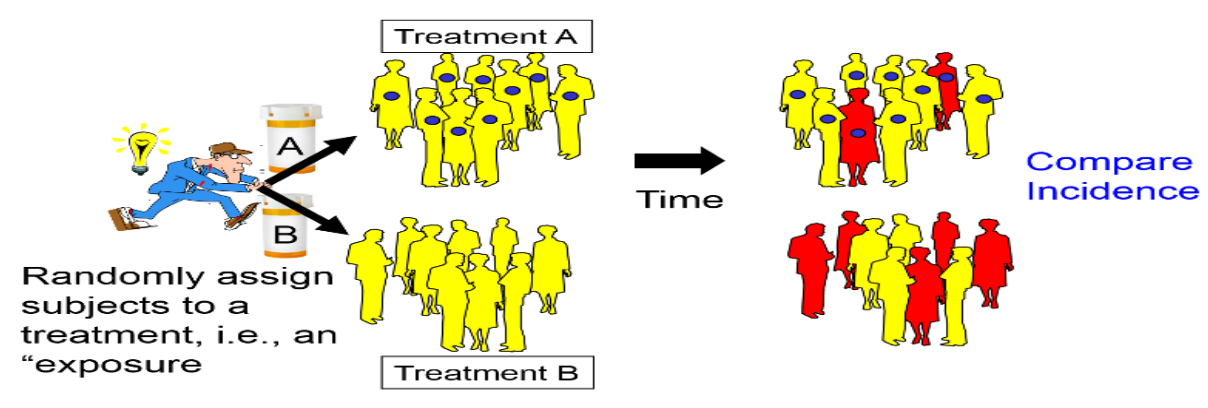

Figure 6: shows how interventional studies 


\subsection{Conclusion}

All interventional, case control, cohort control, and descriptive studies are known as observational studies in epidemiology. They are important in epidemiological surveillance and investigation. Interventional studies are used to assess the effect of any intervention provided for certain outcome within population and in interventional studies mentioned above clear understanding on this type of studies were provided. Case control studies are retrospective and consider outcome to measure the exposure. Cohort control considers the exposure to

measure the outcome and descriptive studies show the frequencies of both exposure and outcome in human population, this kind of study is used in prevalence, incidences and other measures of diseases frequencies.

2. Validity of a diagnostic test (sensitivity, specificity, Positive Predictive Value, Negative Predictive Value)

\subsection{Sensitivity and specificity}

\section{Truth}

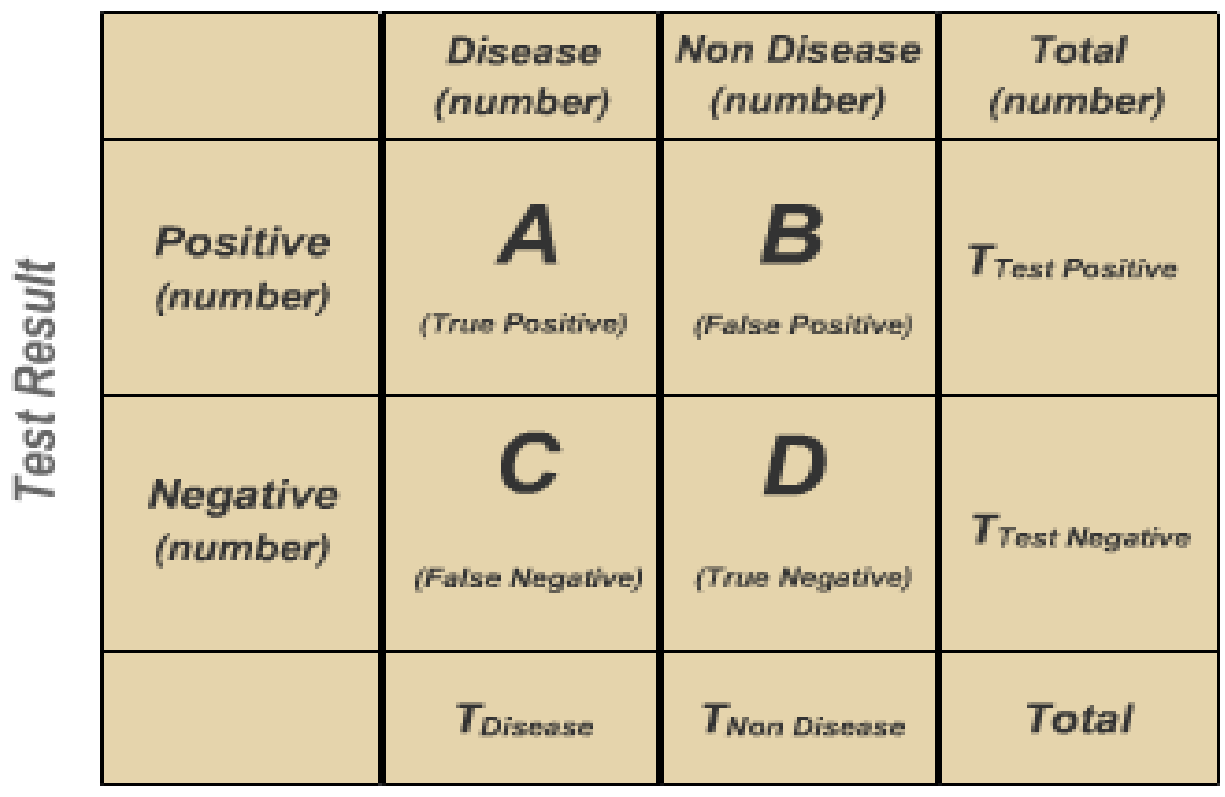

In the above example as indicated in the table, two columns of the above table mention the actual situation of the outcome, diseased people or nondiseased people. The rows of the table mention the results of the diagnostic test from laboratory, either positive or negative test. In diagnosis of disease, the test can be positive or negative. In the interpretation of results from diagnosis, the test can be positive when a patient has outcome or not, it can also be negative when the patient has no disease or has the outcome. This leads us to important terms known us sensitivity and specificity.

Taking observation in the table above, cell A is contained by true positives, indicating diseased people in which the test has been positive. In Cell $\mathrm{D}$ indicates condition where the person has no disease and the test confirms. In diagnosis of diseases, the results in diagnosis should be considered if it has minimum number in cell Band C. Cell B indicates individuals without disease but for whom the test results indicate the positive test, meaning that an individual is diseased. These are known as false positives tests. Cell $\mathrm{C}$ contains the false negatives.

If these results are done to collect data from a population-based study, prevalence of disease is calculated as shown below:

Prevalence of Disease $=\frac{T \text { disease }}{\text { Total }} \times 100$

The population used in certain study influence in calculating the prevalence of the disease in population studied.

Sensitivity is known as the probability of those with the disease who will have positive test. Sensitivity: $=\frac{A}{A+C} \times 100$ 
Specificity is the rate or fraction of those without disease who will have a negative test result:

$$
\text { Specificity: } \mathrm{X}=\frac{\mathrm{D}}{\mathrm{D}+\mathrm{B}} \mathrm{X} 100
$$

\subsection{Positive Predictive Value and Negative Predictive Value}

Different questions may come on both sides of the clinician the patient: what is the probability of reality that a patient with positive test results really has the outcome of interest? If we consider the above table, what is the chance of being in cell $\mathrm{A}$ comparing to cell $\mathrm{B}$

Positive predictive value: If the result indicated positive test, then what is the chance that the patient really has the outcome?"

$$
\mathrm{PPV}=\frac{\mathrm{A}}{\mathrm{A}+\mathrm{B}} \mathrm{X} 100
$$

Negative Predictive Value: If the result indicated negative test, then what is the chance that the patient does not really have the outcome?"

$$
\mathrm{NPV}=\frac{\mathrm{D}}{\mathrm{D}+\mathrm{C}} \times 100
$$

\section{Acknowledgement}

The author acknowledges all authors of reviewed articles found in this article and all contributors of this article are acknowledged

\section{References}

1 Coggon, D., Rose, G. and Barker, D.J.P. Epidemiology for the Uninitiated, London, British Medical Journal Books 2003.

2 Bhopal, R. Concepts of Epidemiology: An Integrated Introduction to the Ideas, Theories, Principles and Methods of Epidemiology. Oxford, Oxford University Press 2002.

3 Erens, B., Primatesta, P. and Prior, G. Health Survey for England 1999: The Health of Minority Ethnic Groups '99, London, The Stationery Office 2001.

4 Tam CC. Newspeak for epidemiologists. J Epidemiol Community Health 2007; 61(3): 178-179 [PMID: 17325389 PMCID: PMC2652901 DOI: 10.1136/jech.2006.055848]

5 Miller, R.E. Epidemiology for Health Promotion and Disease Prevention Professionals, New York, The Haworth Press 2002.
6 Howden-Chapman P, Kawachi I. Paths to and from poverty in late 19th century novels. $J$ Epidemiol Community Health 2006; 60(2): 102-107 [PMID: 16415257 PMCID: PMC2566135 DOI: 10.1136/jech.2005.038794]

7 Stewart, A. Basic Statistics and Epidemiology: A Practical Guide, Oxford, Radcliffe Medical Press 2002

8 Morabia A. Should an epidemiologist also have been awarded the 2008 Nobel Prize in Medicine? J Epidemiol Community Health 2009; 63(3): 177-178 [PMID: 19218253 DOI: 10.1136/jech.2008.083519]

9 Enrique F. Schisterman. Novel Study Designs and Their Application in Reproductive and Perinatal Epidemiology, 2011. DOI:10.1093/acprof:oso/9780195387902.003. $\underline{0102}$

10 Dube L, Van den Broucke S, Housiaux M, Dhoore W, Rendall-Mkosi K. Type 2 diabetes self-management education programs in high and low mortality developing countries: a systematic review. Diabetes Educ 2015; 41(1): 69-85 [PMID: 25392297 DOI: 10.1177/0145721714558305]

11 Rodrigues L, Kirkwood BR. Case-control designs in the study of common diseases: updates on the demise of the rare disease assumption and the choice of sampling scheme for controls. Int J Epidemiol 1990; 19(1): 205 213 [PMID: 2190942]

12 Mandal, J. A Study of Maternal and Perinatal Outcome in Women with and without Gestational Diabetes Mellitus According to International Association of Diabetes and Pregnancy Study Group (IADPSG) Criteria. Journal of Medical Science And clinical Research 2018, 8(6).

13 Austin H, Drews C, Partridge EE. A casecontrol study of endometrial cancer in relation to cigarette smoking, serum estrogen levels, and alcohol use. Am J Obstet Gynecol 1993; 169(5): 1086-1091 [PMID: 8238164]

14 Ransohoff DF, Feinstein AR. Problems of spectrum and bias in evaluating the efficacy of diagnostic tests. $N$ Engl J Med 1978; 299(17): 926-930 [PMID: 692598 DOI: $\underline{10.1056 / N E J M 197810262991705]}$ 
15 Akhmedkhanov A, Zeleniuch-Jacquotte A, Toniolo P. Role of exogenous and endogenous hormones in endometrial cancer: review of the evidence and research perspectives. Ann $\boldsymbol{N} \boldsymbol{Y}$ Acad Sci 2001; 943: 296-315 [PMID: 11594550]

16 Ando M, Wakai K, Seki N, Tamakoshi A, Suzuki K, Ito Y, Nishino Y, Kondo T, Watanabe Y, Ozasa K, Ohno Y, Group JS. Attributable and absolute risk of lung cancer death by smoking status: findings from the Japan Collaborative Cohort Study. Int J Cancer 2003; 105(2): 249-254 [PMID: 12673687 DOI: $10.1002 / \mathrm{ijc} .11043]$

17 Millennium Cohort Study. Millennium Cohort

Study: First Survey, 2001-2003 (5th edn), 2006

18 Colleen R. Implication of Climate Change on Malaria in Karnataka, India. Senior Honors Thesis in Environmental Studies, Brown University 2002, India, 103

19 Pearce N. The four basic epidemiologic study types. J Epidemiol Biostat 1998, vol. 3(pg.171-77)

20 Brosens I, Pijnenborg R, Vercruysse L, Romero R. The "Great Obstetrical Syndromes" are associated with disorders of deep placentation. Am J Obstet Gynecol 2011; 204(3): $\quad$ 193-201 DOI: 10.1016/j.ajog.2010.08.009

21 Bouvier-Colle MH, Mohangoo AD, Gissler M, Novak-Antolic Z, Vutuc C, Szamotulska K, Zeitlin J, Euro-Peristat Scientific C. What about the mothers? An analysis of maternal mortality and morbidity in perinatal health surveillance systems in Europe. BJOG 2012; 119(7): 880-889; discussion 890 DOI: 10.1111/j.1471-0528.2012.03330.x

22 Bossuyt PM, Reitsma JB, Bruns DE, Gatsonis CA, Glasziou PP, Irwig LM, Moher D, Rennie D, de Vet HC, Lijmer JG, Standards for Reporting of Diagnostic A. The STARD statement for reporting studies of diagnostic accuracy: explanation and elaboration. Clin Chem 2003; 49(1): 7-18 [PMID: 12507954]

23 Sobue T, Yamamoto S, Hara M, Sasazuki S, Sasaki S, Tsugane S, Center JSGJPH. Cigarette smoking and subsequent risk of lung cancer by histologic type in middle-aged Japanese men and women: the JPHC study. Int J Cancer 2002; 99(2): 245-251 [PMID: 11979440 DOI: $10.1002 / \mathrm{ijc} .10308]$ 